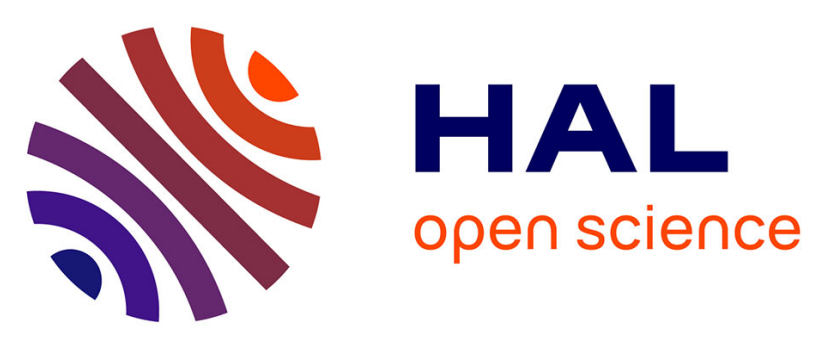

\title{
Fetal ECG extraction by extended state Kalman filtering Based on Single-Channel Recordings
}

\author{
Mohammad Niknazar, Bertrand Rivet, Christian Jutten
}

\section{To cite this version:}

Mohammad Niknazar, Bertrand Rivet, Christian Jutten. Fetal ECG extraction by extended state Kalman filtering Based on Single-Channel Recordings. IEEE Transactions on Biomedical Engineering, 2013, 60 (5), pp.1345-1352. 10.1109/TBME.2012.2234456 . hal-00831928

\section{HAL Id: hal-00831928 https://hal.science/hal-00831928}

Submitted on 8 Jun 2013

HAL is a multi-disciplinary open access archive for the deposit and dissemination of scientific research documents, whether they are published or not. The documents may come from teaching and research institutions in France or abroad, or from public or private research centers.
L'archive ouverte pluridisciplinaire HAL, est destinée au dépôt et à la diffusion de documents scientifiques de niveau recherche, publiés ou non, émanant des établissements d'enseignement et de recherche français ou étrangers, des laboratoires publics ou privés. 


\title{
Fetal ECG Extraction by Extended State Kalman Filtering Based on Single-Channel Recordings
}

\author{
M. Niknazar, B. Rivet, and C. Jutten, IEEE Fellow
}

\begin{abstract}
In this paper, we present an extended nonlinear Bayesian filtering framework for extracting ECGs from a singlechannel as encountered in the fetal ECG extraction from abdominal sensor. The recorded signals are modeled as the summation of several ECGs. Each of them is described by a nonlinear dynamic model, previously presented for the generation of a highly realistic synthetic ECG. Consequently, each ECG has a corresponding term in this model and can thus be efficiently discriminated even if the waves overlap in time. The parameter sensitivity analysis for different values of noise level, amplitude and heart rate ratios between fetal and maternal ECGs shows its effectiveness for a large set of values of these parameters. This framework is also validated on the extractions of fetal ECG from actual abdominal recordings, as well as of actual twin magnetocardiograms.
\end{abstract}

Index Terms-fetal ECG extraction, twin MCGs extraction, extended Kalman filtering, nonlinear Bayesian filtering, modelbased filtering.

\section{INTRODUCTION}

Since the first demonstration of the fetal electrocardiogram (fECG) carried out in 1906 by Cremer [1], various methods for fECG monitoring have been proposed to obtain information about the heart status. The fECG can be measured by placing electrodes on the mother's abdomen. However, it has very low power and is mixed with several sources of noise and interference. Nevertheless, the main contamination is the maternal electrocardiogram (mECG) [2]. As a result, the basic problem is to extract the fECG signal from the mixture of mECG and fECG signals, where the interfering mECG is a much stronger signal. According to the review [3], existing fECG extraction approaches in literature can be categorized by their methodologies, which include linear or nonlinear decomposition and adaptive filtering.

Linear or nonlinear decomposition methods are common approaches in which, single or multi-channel recordings are decomposed into different components using suitable basis functions. Linear decomposition methods use either fixed basis functions (e.g., wavelets [4]), or data-driven basis functions (e.g., singular vectors [5]). This limits performance of decomposition in nonlinear or degenerate mixtures of signal and noise [3]. Blind or semi-blind source separation, which are categorized as linear decomposition approach, have also been used for fECG extraction [6], [7]. These methods are based on the assumption of independent components (or more generally independent subspaces [8] or partitions [9]) for the maternal and fetal signals, or of the existence of some temporal structure

The authors are with the GIPSA-lab (UMR CNRS 5216), University of Grenoble, Grenoble, France (email: \{mohammad.niknazar, bertrand.rivet, christian.jutten\}@gipsa-lab.grenoble-inp.fr). C. Jutten is also with the Institut Universitaire de France. for the desired signals [10], [11], [12]. In [13], [14], wavelet decomposition was also combined with blind source separation for extracting and denoising fECG signals. In another recent work, a new technique was proposed to fasten traditional Independent Component Analysis (ICA) method [15]. In blind source separation methods it is usually assumed that signals and noises are mixed in a stationary and linear manner. However, fECG and other interferences and noises are not always stationary mixed and linearly separable [16].

Nonlinear transforms have been also used for mECG cancellation and fECG extraction. In these methods, constructed phase space of noisy signal and of its delayed versions is smoothed using conventional or Principal Component Analysis (PCA) smoothers [17]. The samples are then transferred back to the time-domain representation. Although these methods are interesting since they are applicable to as few as one single maternal abdominal channel, the selection of the required timelags for constructing phase space representation is empirical and the important inter-beat variations of the cardiac signals can be wiped-out during the state-space smoothing. Moreover, they demand higher computational complexity in comparison to linear methods, and the correct embedding dimension can change as the noise statistics change [3].

Adaptive filtering is another common approach for mECG cancellation and fECG extraction [18]. The conventional adaptive filtering is based on training an adaptive filter for either removing the mECG using one or several maternal reference channels [18], [19], or directly training the filter for extracting the fetal QRS waves [20], [21]. However, existing adaptive filtering methods for $\mathrm{mECG}$ artifact removal, either require a reference mECG channel that is morphologically similar to the contaminating waveform or require several linearly independent channels to roughly reconstruct any morphologic shape from the references [18]. Both of these approaches are practically inconvenient and with limiting performance, because the morphology of the mECG contaminants highly depends on the electrode locations and it is not always possible to reconstruct the complete $\mathrm{mECG}$ morphology from a linear combination of the reference electrodes [3]. Practically, it has been shown that for fECG extraction, blind source separation methods outperform adaptive filters [22]. An important advantage of spatial filtering over conventional adaptive filters is their ability to separate $\mathrm{mECG}$ and fECG with temporal overlap but it often requires more than two sensors.

The Kalman filtering framework, which can be considered as a member of the general class of adaptive filters, is a promising approach for both mECG cancellation and fECG enhancement. In [16], [23] a set of state-space equations was used to model the temporal dynamics of ECG signals, for 
designing a Bayesian filter for ECG denoising. This Bayesian filter framework was used in [16, p. 50] to extract fECG from single channel mixture of mECG and fECG. However, as it is mentioned in [16], the filter fails to discriminate between the maternal and fetal components when the mECG and fECG waves fully overlap in time. The reason is that when mECG is being estimated, fECG and other components are supposed to be Gaussian noise. However, this assumption is not true, especially when mECG and fECG waves fully overlap in time it is difficult for the filter to follow desired ECG.

Clinical monitoring of fetal cardiac activity is usually based on a small number of electrodes located on mother's abdomen, and on a sound sensitive sensor. In such a context, in the present work, we wonder what performance can be obtained with only one electrode, by using a refined model of the signal recorded on the unique electrode: the model will explicitly take into account that the signal is the superposition of a few ECG signals. The rest of this paper is organized as follows. In section II equations and theory supporting our proposed method including the Bayesian filtering theory and dynamic ECG model are described. In section III results of the proposed method applied on different data and discussion about the results are presented. Finally, our conclusion is stated in section IV.

\section{Methodology}

\section{A. EKF Framework for ECG Extraction}

The goal of Kalman Filter (KF) is to estimate the state of a discrete-time controlled process. Consider a state vector $\mathbf{x}_{k+1}$ governed by a nonlinear stochastic difference equation with measurement vector $\mathbf{y}_{k+1}$ at time instant $k+1$ :

$$
\left\{\begin{array}{l}
\mathbf{x}_{k+1}=f\left(\mathbf{x}_{k}, \mathbf{w}_{k}, k+1\right) \\
\mathbf{y}_{k+1}=h\left(\mathbf{x}_{k+1}, \mathbf{v}_{k+1}, k+1\right)
\end{array}\right.
$$

where the random variables $\mathbf{w}_{k}$ and $\mathbf{v}_{k}$ represent the process and measurement noises, with associated covariance matrices $Q_{k}=E\left\{\mathbf{w}_{k} \mathbf{w}_{k}^{T}\right\}$ and $R_{k}=E\left\{\mathbf{v}_{k} \mathbf{v}_{k}^{T}\right\}$. The Extended Kalman Filter (EKF) is an extension of the standard KF to nonlinear systems $f(\cdot)$ and $h(\cdot)$, which linearizes about the current mean and covariance [24]. In order to improve the estimations, EKF can be followed by a backward recursive smoothing stage leading to the Extended Kalman Smoother (EKS). However, since EKS is a non causal method, it can not be applied online but it is useful if a small lag in the processing is allowed.

In this work, a synthetic dynamic ECG model [25] is used to extract fECG from mixture of an $\mathrm{mECG}$, one (or more) fECG(s) and other signals considered as noises. In polar coordinates [23], one ECG signal can be expressed as the sum of five Gaussian functions defined by their peak amplitude, width and center, denoted $\alpha_{i}, b_{i}$ and $\psi_{i}$, respectively: $z(\theta)=$ $\sum_{i \in \mathcal{W}} \alpha_{i} \exp \left(-\left(\theta-\psi_{i}\right)^{2} /\left(2 b_{i}^{2}\right)\right)$. Each Gaussian function thus models one of the five waves $\mathcal{W}=\{P, Q, R, S, T\}$ of a heart beat. The state vector in equation (1) is defined by the phase $\theta$ and the amplitude $z$ of the ECG: $\mathbf{x}_{k}=\left[\theta_{k}, z_{k}\right]^{T}$. Assuming a small sampling period $\delta$, the state noise $\eta_{k}$, and defining $\mathbf{w}_{k}$

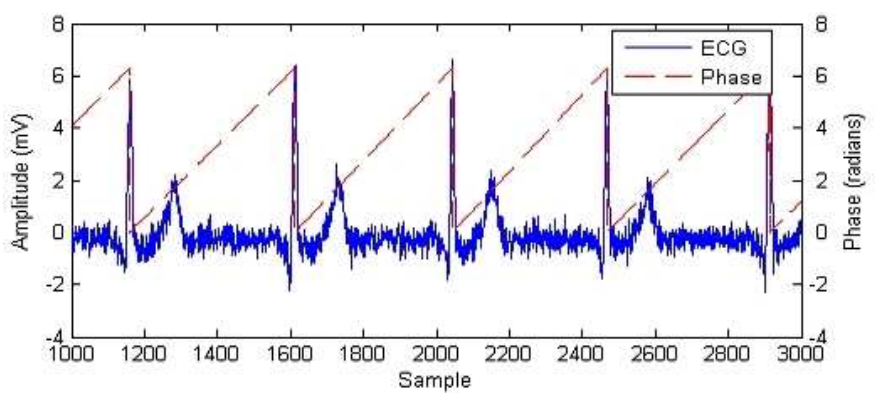

Figure 1. Illustration of the phase assignment approach on one ECG.

as $\left[0, \eta_{k}\right]^{T}$, the state process $f(\cdot)$ is

$$
\begin{aligned}
& \theta_{k+1}=\left(\theta_{k}+\omega \delta\right) \bmod (2 \pi), \\
& z_{k+1}=-\sum_{i \in \mathcal{W}} \frac{\alpha_{i} \Delta \theta_{i, k} \omega \delta}{b_{i}^{2}} \exp \left(-\frac{\Delta \theta_{i, k}^{2}}{2 b_{i}^{2}}\right)+z_{k}+\eta_{k},
\end{aligned}
$$

where $\omega$ is the phase increment, $\Delta \theta_{i, k}=\left(\theta_{k}-\psi_{i}\right) \bmod (2 \pi)$. From the ECG, one can define the observed phase $\phi_{k}$ by a linear time wrapping of the R-R time intervals into $[0,2 \pi)$ (Figure 1). The measurement process $h(\cdot)$ is finally defined as $\mathbf{y}_{k+1}=\mathbf{x}_{k+1}+\mathbf{v}_{k+1}$, where $\mathbf{y}_{k+1}=\left[\phi_{k+1}, s_{k+1}\right]^{T}$.

The ECGs composing the observed mixture can be estimated by recursively applying the described EKF: at each step, one ECG is extracted according to a deflation procedure. In case of a mixture of mECG and one fECG, the first step extracts, from the raw recording, the dominant ECG (often the mECG) considering the concurrent ECG (resp. fECG) and other noises as a unique Gaussian noise. After subtracting the dominant ECG from the original signal, the second step is the extraction of fECG from the residual signal. This procedure is referred to as sequential EKF or EKS (seq-EKF or seq-EKS). In this recursive extraction, during the first step, the concurrent ECG (i.e. fECG) and additional noise are modeled by Gaussian noises $\mathbf{v}_{k}$ and $\mathbf{w}_{k}$, which is not a very relevant assumption. In fact, although this assumption may be acceptable when there are not strong artifacts interfering with the ECG, it is no longer accurate when other ECG artifacts are considerable (i.e. at the first step) since the noise is no longer normally distributed. In addition, concurrent ECGs can be confused with dominant ECG when their waves (especially QRS complexes) fully overlap in time. Meanwhile, resultant inaccuracies, which are generated by the previous steps of the ECG extraction, will propagate to the next steps while the residuals are computed.

\section{B. Extension to multiple ECGs: extended state EKF}

In this paper the dynamic equations (2) and (3) are extended for simultaneously modeling $N$ ECGs mixed in a single observation. The related extended state vector $\mathbf{x}_{k}=$ 


$$
\begin{aligned}
& {\left[\theta_{k}^{(1)}, z_{k}^{(1)}, \ldots, \theta_{k}^{(N)}, z_{k}^{(N)}\right]^{T} \text { is thus defined by }} \\
& \left\{\begin{aligned}
\theta_{k+1}^{(1)}= & \left(\theta_{k}^{(1)}+\omega^{(1)} \delta\right) \bmod (2 \pi) \\
z_{k+1}^{(1)}= & -\sum_{i \in \mathcal{W}_{1}} \frac{\alpha_{i}^{(1)} \omega^{(1)} \delta}{b_{i}^{(1)^{2}}} \Delta \theta_{i, k}^{(1)} \exp \left(-\frac{\Delta \theta_{i, k}^{(1)^{2}}}{2 b_{i}^{(1)^{2}}}\right) \\
& \quad+z_{k}^{(1)}+\eta_{k}^{(1)}
\end{aligned} \quad \begin{array}{rl}
\vdots \\
\theta_{k+1}^{(N)}= & \left(\theta_{k}^{(N)}+\omega^{(N)} \delta\right) \bmod (2 \pi) \\
z_{k+1}^{(N)}= & -\sum_{i \in \mathcal{W}_{N}} \frac{\alpha_{i}^{(N)} \omega^{(N)} \delta}{b_{i}^{(N)^{2}}} \Delta \theta_{i, k}^{(N)} \exp \left(-\frac{\Delta \theta_{i, k}^{(N)^{2}}}{2 b_{i}^{(N)^{2}}}\right) \\
& +z_{k}^{(N)}+\eta_{k}^{(N)}
\end{array}\right.
\end{aligned}
$$

where each $\left[\theta_{k}^{(i)}, z_{k}^{(i)}\right]^{T}$ is related to one of the ECGs. Finally, the measurement process leads to express the measurement vector $\mathbf{y}_{k+1}=\left[\phi_{k+1}^{(1)}, \cdots, \phi_{k+1}^{(N)}, s_{k+1}\right]^{T}$ as

$$
\left\{\begin{array}{l}
\phi_{k+1}^{(n)}=\theta_{k+1}^{(n)}+v_{k+1}^{(n)}, \\
s_{k+1}=\sum_{n=1}^{N} z_{k+1}^{(n)}+v_{k+1}^{(N+1)} .
\end{array} \quad \forall n \in\{1, \cdots, N\}\right.
$$

This extended state Kalman filtering procedure is referred to as parallel EKF or EKS (par-EKF, or par-EKS, respectively). As shown in the results section (Section IIII), this par-EKF or par-EKS is more accurate to extract fECG from abdominal sensors than the seq-EKF or seq-EKF. Indeed, in the proposed method all ECGs are jointly modeled by dynamic states so that only the state and measurement noise vectors are assumed to be normally distributed. Moreover, the extended state par-EKF fully models overlapping waves of several ECGs. Finally, the state and observation noises, $\eta_{k}^{n}$ and $v_{k}^{n}$, respectively, allow to fit some variabilities of the ECG shapes. Even if the model do not fit too large variations (for example due to arrythmia), but an inspection of the residue will reveal these abnormal beats.

\section{Model parameters estimation}

The proposed par-EKF and par-EKS lie on several state parameters $\left\{\alpha_{i}^{(n)}, b_{i}^{(n)}, \psi_{i}^{(n)}\right\}_{i \in \mathcal{W}_{n}}, \forall n \in\{1, \ldots, N\}$. The procedure described below is an extension of the single ECG parameter estimation [23].

The parameters estimation procedure first needs the R-peaks detection for all ECGs to perform the time-wrapping of the $\mathrm{R}-\mathrm{R}$ intervals into $[0,2 \pi)$ to define $\phi_{k}^{(n)}$. The R-peaks are found from a peak search in windows of length $T$, where $T$ corresponds to the R-peak period calculated from approximate ECG beat-rate. R-peaks with periods smaller than $\frac{T}{2}$ or larger than $T$ are not detected. Although maternal R-peaks are easily detectable from the mixture, fetal R-peaks detection is more complex due to its lower amplitude than mECG. Therefore, a rough estimation of fECG is obtained by using the seqEKF algorithm, which now allows to detect easily the fetal R-peak\$. Now, for each ECG, each beat (defined by the signals between two consecutive R-peaks) is time wrapped into $[0,2 \pi)$. The average of the ECG waveform is obtained

\footnotetext{
${ }^{1}$ In practice, one could also use a sound sensor to have a reliable R-peak detector. In this case, even if there exists a delay, it does not impact the method, since it can be synchronized.
}

by the mean of all time-wrapped beats, for all phases between 0 and $2 \pi$. Finally, by using a nonlinear least-squares approach [26], the best estimate of the parameters in the minimum mean square error (MMSE) sense is found.

\section{RESUlts AND Discussions}

Both synthetic and actual data have been used to study performance of the proposed method. In the first subsection, quantitative results coming from simulations and influence of the main parameters of mixed ECGs on performance of the method has been studied. They will present the conditions in which, the proposed method is efficient. In the second subsection the effectiveness of the method on actual data has been examined.

\section{A. Experimental Performance Analysis on Synthetic Data}

Since there is neither ground truth nor golden standard on single channel recording, it is important to provide quantitative performance with simulations to validate the behavior of the proposed method. In order to do so, realistic synthetic mixtures of $\mathrm{mECG}$ and fECG with white Gaussian noise have been generated for different situations and the proposed method has been applied on them to extract mECG and fECG.

Synthetic mECG and fECG used in this study are based on a three-dimensional canonical model of the single dipole vector of the heart, proposed in [27] and inspired by the singlechannel ECG dynamic model presented in [25]. Sampling frequency is set to $500 \mathrm{~Hz}$ and signals include 20,000 samples. The main parameters that can affect the mixtures are input noise power, ratio between amplitudes of fECG and mECG, and ratio between fetal and maternal heart rates. In order to investigate the performance of the proposed method hundred trials were carried out under each value of these parameters. In the output, estimated mECG and fECG signals, $\hat{s}_{m}$ and $\hat{s}_{f}$, are assumed to be the sum of mECG, fECG and noise, such that:

$$
\begin{aligned}
& \hat{s}_{m}=\alpha_{1} s_{m}+\alpha_{2} s_{f}+\alpha_{3} n, \\
& \hat{s}_{f}=\beta_{1} s_{m}+\beta_{2} s_{f}+\beta_{3} n,
\end{aligned}
$$

where coefficients $\alpha_{1}, \alpha_{2}, \alpha_{3}, \beta_{1}, \beta_{2}$, and $\beta_{3}$, have to be estimated and $s_{m}, s_{f}$, and $n$ denote $\mathrm{mECG}$, fECG and noise, respectively. In order to estimate the coefficients, $s_{m}, s_{f}$, and $n$ are assumed to be orthogonal, i.e., decorrelated. The orthogonality principle states that an estimator $\hat{s}$ achieves MMSE if and only if $E\left\{(\hat{s}-s)^{T} \hat{s}\right\}=0$. Satisfaction of this criteria leads to:

$$
\begin{array}{lll}
\hat{\alpha}_{1}=\frac{E\left(\hat{s}_{m}^{T} s_{m}\right)}{E\left(s_{m}^{T} s_{m}\right)}, & \hat{\alpha}_{2}=\frac{E\left(\hat{s}_{m}^{T} s_{f}\right)}{E\left(s_{m}^{T} s_{f}\right)}, & \hat{\alpha}_{3}=\frac{E\left(\hat{s}_{m}^{T} n\right)}{E\left(s_{m}^{T} n\right)}, \\
\hat{\beta}_{1}=\frac{E\left(\hat{s}_{f}^{T} s_{m}\right)}{E\left(s_{f}^{T} s_{m}\right)}, & \hat{\beta}_{2}=\frac{E\left(\hat{s}_{f}^{T} s_{f}\right)}{E\left(s_{f}^{T} s_{f}\right)}, & \hat{\beta}_{3}=\frac{E\left(\hat{s}_{f}^{T} n\right)}{E\left(s_{f}^{T} n\right)} .
\end{array}
$$

In a successful estimation, contribution of desired ECG in output should be much more than contribution of undesired ECG and noise. In other words, in extraction of fECG the power of $\beta_{2} s_{f}$ should be much larger than power of $\beta_{1} s_{m}+$ $\beta_{3} n$, which means the contribution of $\mathrm{mECG}$ and noise is very 


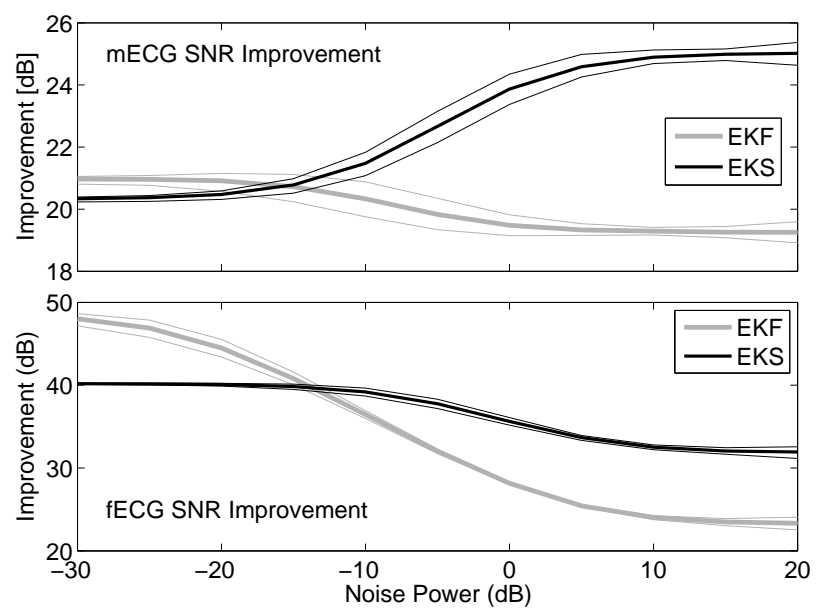

Figure 2. Mean SNR improvement results of the EKF and EKS against input noise power (bold lines). Upper and lower borders (thin lines) present maximum and minimum, respectively.

low in the fECG estimate. In the same manner, the power of $\alpha_{1} s_{m}$ should be much larger than power of $\alpha_{2} s_{f}+\alpha_{3} n$ in $\mathrm{mECG}$ extraction. In order to quantize contribution of the desired ECG in the output, output Signal to Noise Ratio (SNR) for maternal and fetal ECG are defined as:

$$
\begin{aligned}
& S N R_{m_{\text {out }}}=\frac{\hat{\alpha}_{1}^{2} P_{s_{m}}}{\hat{\alpha}_{2}^{2} P_{s_{f}}+\hat{\alpha}_{3}^{2} P_{n}}, \\
& S N R_{f_{\text {out }}}=\frac{\hat{\beta}_{2}^{2} P_{s_{f}}}{\hat{\beta}_{1}^{2} P_{s_{m}}+\hat{\beta}_{3}^{2} P_{n}} .
\end{aligned}
$$

where $P_{s_{m}}, P_{s_{f}}$, and $P_{n}$ denote power of mECG, fECG, and noise, respectively. Output SNR is now compared to input SNR to investigate performance of desired ECG extraction. Input SNRs are defined as:

$$
S N R_{m_{i n}}=\frac{P_{s_{m}}}{P_{s_{f}}+P_{n}} \quad \text { and } \quad S N R_{f_{i n}}=\frac{P_{s_{f}}}{P_{s_{m}}+P_{n}}
$$

Input Signal to Interference Ratio (SIR) and output SIR are also defined as:

$$
\begin{aligned}
& S I R_{m_{\text {in }}}=\frac{P_{s_{m}}}{P_{s_{f}}}, \quad S I R_{f_{\text {in }}}=\frac{P_{s_{f}}}{P_{s_{m}}}, \\
& S I R_{m_{\text {out }}}=\frac{\hat{\alpha}_{1}^{2} P_{s_{m}}}{\hat{\alpha}_{2}^{2} P_{s_{f}}}, \quad S I R_{f_{\text {out }}}=\frac{\hat{\beta}_{2}^{2} P_{s_{f}}}{\hat{\beta}_{1}^{2} P_{s_{m}}} .
\end{aligned}
$$

1) SNR Analysis: Figure 2 shows SNR improvement results of EKF and EKS over a wide range of input noise power. The SNR improvement in $\mathrm{dB}$ is defined as the output SNR of the filter minus the input SNR. In all trials, power of $\mathrm{mECG}$ signals is normalized to $1(0 \mathrm{~dB})$ and the ratio of amplitudes of fECG and mECG is 0.3. Maternal and fetal heart rates are set to $1.1 \mathrm{~Hz}$ and $2 \mathrm{~Hz}$, respectively. Moreover, in order to have more realistic signals, mECG and fECG are allowed to have slight random fluctuations $(5 \%)$ in amplitude and duration at each beat. Moreover, initial phases of ECGs are random. As it can be seen in Figure 2, both EKF and EKS successfully improved the SNR for all ranges of the input SNRs. When the mixture is rather noise free (noise power $-30 \mathrm{~dB}$ ) the minimum SNR improvement of fECG is $40 \mathrm{~dB}$, which means efficient
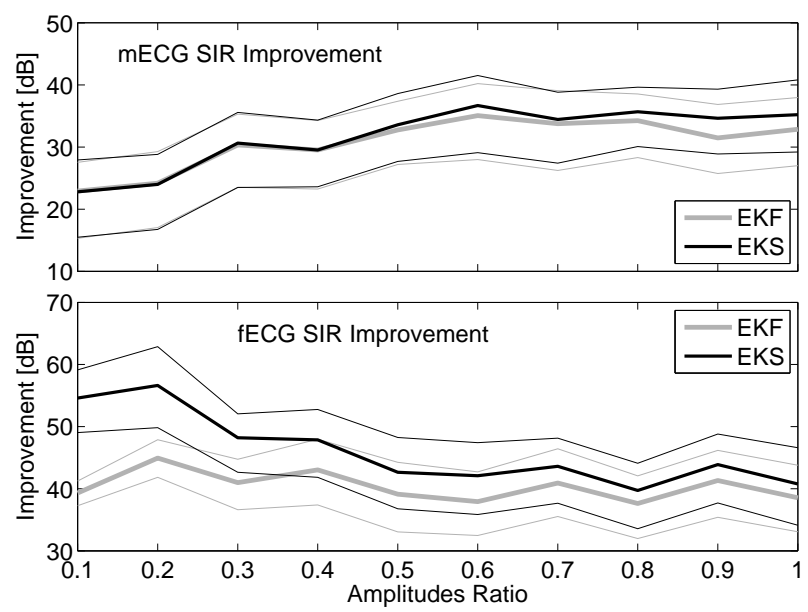

Figure 3. Mean SIR improvement results of the EKF and EKS against amplitude ratio (bold lines). Upper and lower borders (thin lines) present maximum and minimum, respectively.

cancellation of mECG. Nevertheless, even for very noisy mixtures (noise power $20 \mathrm{~dB}$ ), the SNR improvement of fECG remains over $20 \mathrm{~dB}$. According to this figure, EKF is more effective when a rather clean signal is available. However, as power of noise increases, EKS significantly outperforms EKF. As it has been explained in the previous section, the EKS algorithm consists of a forward EKF stage followed by a backward recursive smoothing stage. Therefore, if a rather clean signal is available, the recursive smoothing stage will deteriorate EKF output, because the output is smooth enough and recursive smoothing leads to over-filtering. Conversely, if the signal is very noisy, EKF output is not denoised enough yet. Therefore, recursive smoothing stage can be successfully used to cancel more noise from the signal.

2) Amplitude Ratio Analysis: The basic problem of fECG monitoring is to extract the fECG signal from the mixture of $\mathrm{mECG}$ and fECG signals, where the interfering $\mathrm{mECG}$ is a stronger signal. Therefore, it is necessary to evaluate the performance of the method for different ratios of fECG and $\mathrm{mECG}$ amplitudes. For this purpose, SIR improvement of output signals have been calculated in the range of 0.1 to 1 of amplitude ratio of fECG and mECG. Figure 3 shows SIR improvement results of the EKF and EKS for different values of amplitude ratios. Power of mECG signals are normalized to $1(0 \mathrm{~dB})$ with $5 \%$ random fluctuation, input SNR with respect to (w.r.t.) $\mathrm{mECG}$ is $10 \mathrm{~dB}$, and average maternal and fetal heart rates are $1.1 \mathrm{~Hz}$ and $2 \mathrm{~Hz}$, respectively. As it is seen in Figure 3, although the fetal SIR improvements of both EKF and EKS remain over $30 \mathrm{~dB}$ for all ranges of the amplitude ratios, results of EKS are slightly better.

3) Heart Rate Ratio Analysis: Since fetal heart rate may vary in a wide range [28], the performance of the method was studied on a wide range of $0.3 \mathrm{~Hz}$ to $3.6 \mathrm{~Hz}$ of fetal heart rate. Figure 4 shows SIR improvement results of EKF and EKS. Power of mECG signals are normalized to $1(0 \mathrm{~dB})$ with $5 \%$ random fluctuation and the ratio of amplitudes of fECG and mECG is 0.3. Input SNR w.r.t. mECG is $10 \mathrm{~dB}$, and maternal heart rate is set to $1.1 \mathrm{~Hz}$. In this section, heart 

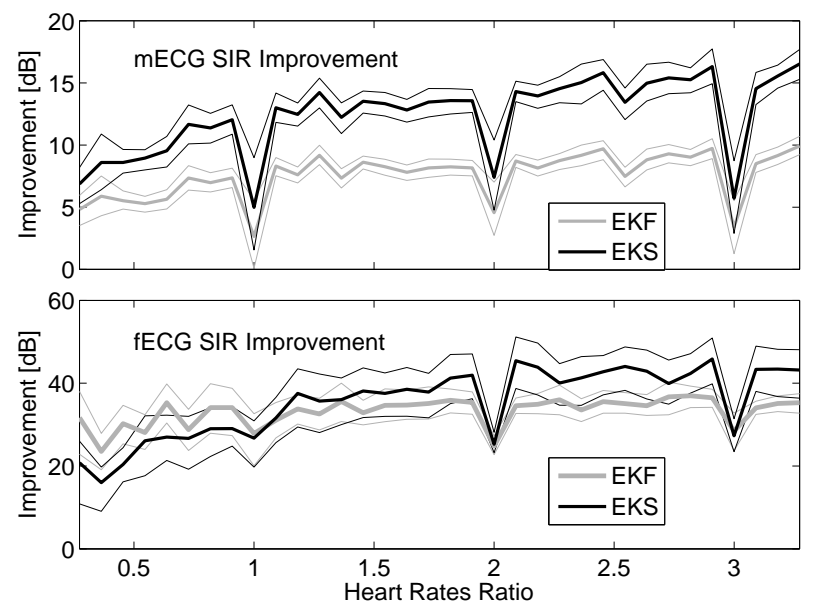

Figure 4. Mean SIR improvement results of the EKF and EKS against heart rate ratio (bold lines). Upper and lower borders (thin lines) present maximum and minimum, respectively.

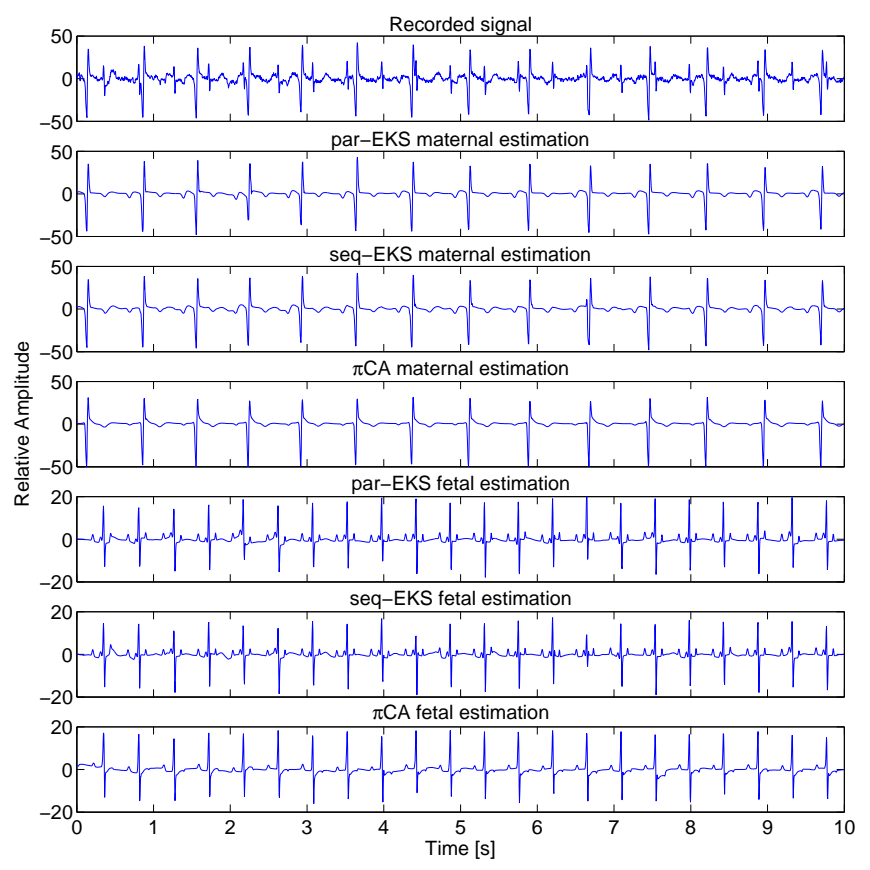

Figure 5. Comparison of fECG extraction by par-EKS, seq-EKS and $\pi \mathrm{CA}$ on the first channel of DaISy data.

rate fluctuations are slighter (1\%) to study harmonic issues more accurately. As expected, SIR improvement diagram has three deep local minima at ratios 1,2 and 3 . The reason is that when main frequencies of mECG and fECG are proportional, the signals overlap more closely in the frequency domain. Therefore, discrimination of $\mathrm{mECG}$ and $\mathrm{fECG}$ is more difficult for these ratios. Nevertheless, these situations are unlikely happening because the heart rates ratio is usually more than 1 and less than 2. Even in these cases, fetal SIR improvement remains over $20 \mathrm{~dB}$. Here again, EKS slightly outperforms EKF.

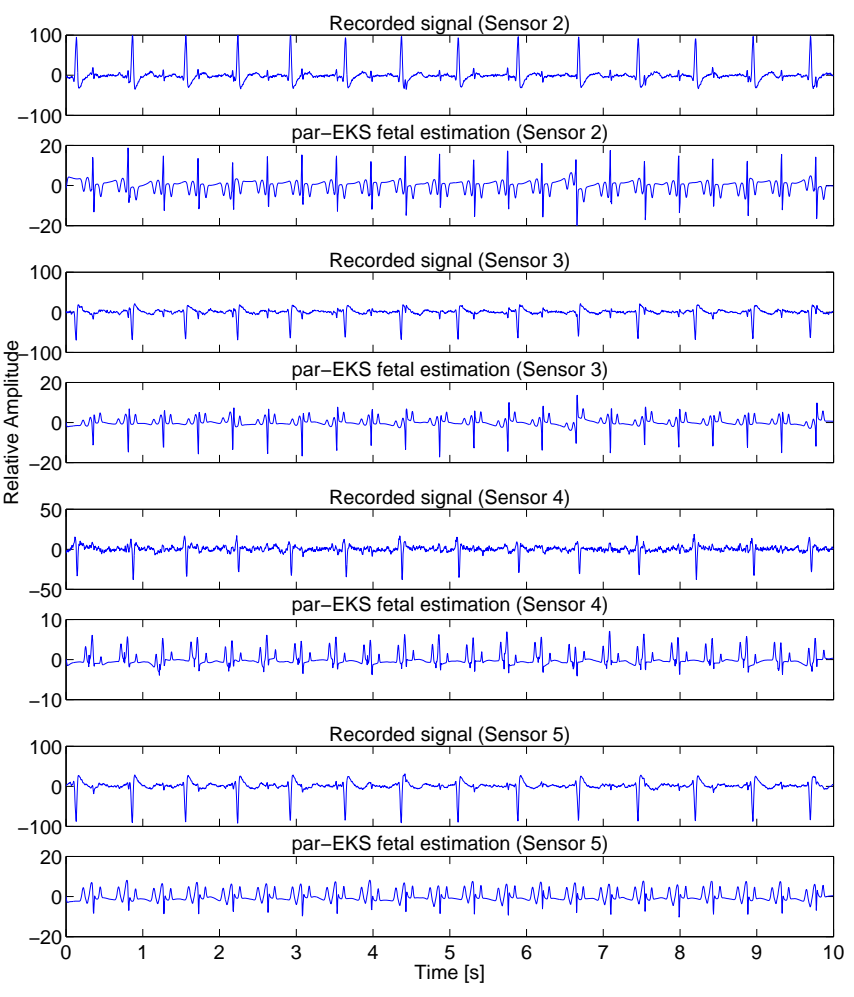

Figure 6. Results of fECG extraction using par-EKS applied on channels 2 to 5 of the DaISy dataset (up to down). Note differences of scales, according to the channels and the fetal estimates.

\section{B. Fetal ECG Extraction on Actual Data}

In the previous subsection, efficiency of the proposed method in fECG extraction for a wide range of possible configurations has been examined using synthetic data. In this subsection, the results of application of the proposed method on actual data are presented.

1) DaISy Database: The DaISy fetal ECG database [29] consists of a single dataset of cutaneous potential recording of a pregnant woman. A total of 8 channels ( 5 abdominal and 3 thoracic) are available, sampled at $250 \mathrm{~Hz}$ and lasting 10 seconds.

Figure 5 presents the results of par-EKS and seq-EKS using the first channel of the dataset. Moreover, the periodic component analysis ( $\pi \mathrm{CA}$ ) [8] using the height channels, which is a multi-channel method, is also included as the golden standard. Results of $\pi \mathrm{CA}$ method are then post-processed via EKS on the best ECG estimate [23]. As already mentioned, unlike seq-EKS, par-EKS does not fail when $\mathrm{mECG}$ and fECG fully overlap in time. This is particularly noticed between $t$ $=6 \mathrm{~s}$ and $\mathrm{t}=7 \mathrm{~s}$ in Figure 5 in which, some parts of fECG signal have been deteriorated during $\mathrm{mECG}$ extraction by the seq-EKS method. On the contrary, the proposed par-EKS jointly models the fECG and $\mathrm{mECG}$, resulting in a better estimate of fECG than seq-EKS. Since par-EKS estimates a single component while $\pi \mathrm{CA}$ can estimate several components (typically one or two), the cosine between subspaces is used, and is equal to 0.92 in this experiment. With a value closed to 1 , these estimates are quite similar. Finally, Figure 6 shows the results of fECG extraction using par-EKS applied on the other 


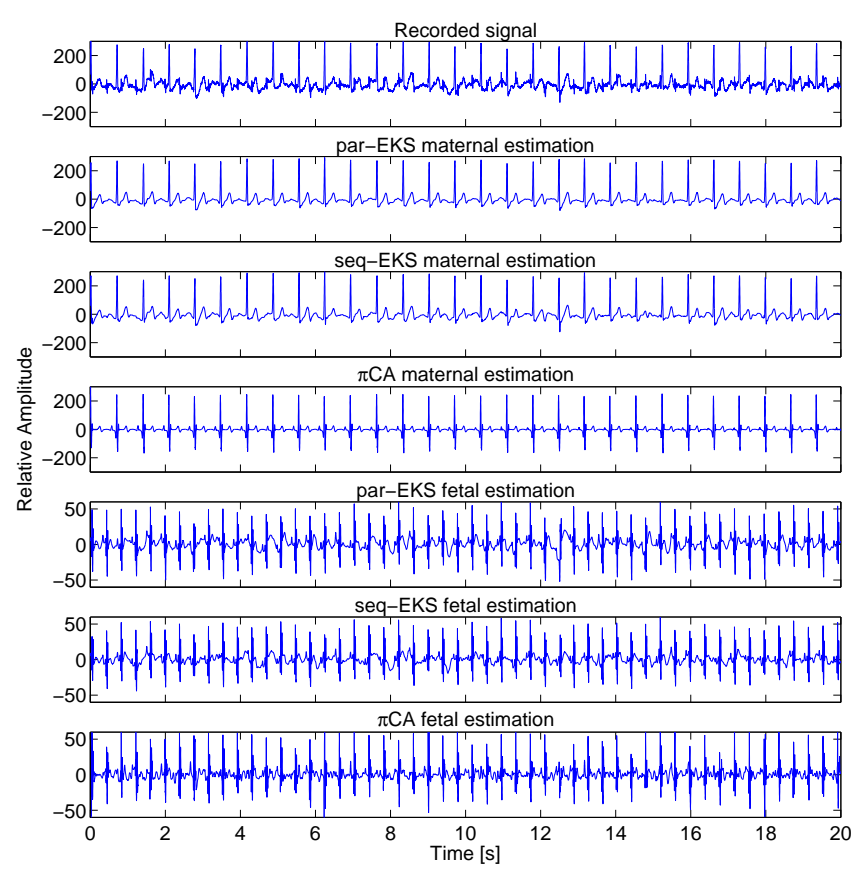

Figure 7. Comparison of fECG extraction by par-EKS, seq-EKS and $\pi \mathrm{CA}$ on ecgca 771 of the PhysioNet database.

abdominal channels of the DaISy dataset. It experimentally proves that par-EKS is able to extract fECG even in illconditionned mixtures, such as channels 4 or 5 .

2) Non-Invasive Fetal Electrocardiogram Database: This database consists of a series of 55 multichannel abdominal fECG recordings, taken from a single subject between 21 to 40 weeks of pregnancy. The recordings include 2 thoracic signals and 3 or 4 abdominal signals. The signals were recorded at $1 \mathrm{kHz}, 16-b i t$ resolution with a bandpass filter $(0.01 \mathrm{~Hz}-100 \mathrm{~Hz})$ and a main notch filter $(50 \mathrm{~Hz})$ [30]. Figure 7 shows results of seq-EKS and par-EKS using channel 3, and $\pi$ CA using all channels of the first 20 s of namely the ecgca 771 dataset. To show the effectiveness of the proposed method in extraction of the fECG at different periods of pregnancy, and from different channel locations, the first 20 s of the mixtures and fetal parEKS outputs of the datasets ecgca274 channel 5, ecgca748 channel 4, and ecgca997 channel 3 are plotted in Figure 8.

3) Twin MCGs Extraction: The proposed method has been principally designed for ECG signals. Nevertheless, due to the morphological similarity of the ECG and the magnetocardiogram (MCG), it is also directly applicable to MCG recordings. In this section, twin fetal cardiac magnetic signals recorded by a SQUID Biomagnetometer system are extracted. The dataset has been recorded in the Biomagnetic Center of the Department of Neurology (Friedrich Schiller University, Jena, Germany) and it consists of 208 channels sampled at $1025 \mathrm{~Hz}$ over 30 minutes.

Figure 9 presents the results of the proposed par-EKS to extract the two fetal MCG signals from a single sensor. A typical channel (indexed 92) of namely the q00002252 dataset has been selected. Even though the multichannel $\pi \mathrm{CA}$ method provides better results in this case than single channel methods (par-EKS or seq-EKS), the proposed par-EKS succeeds to

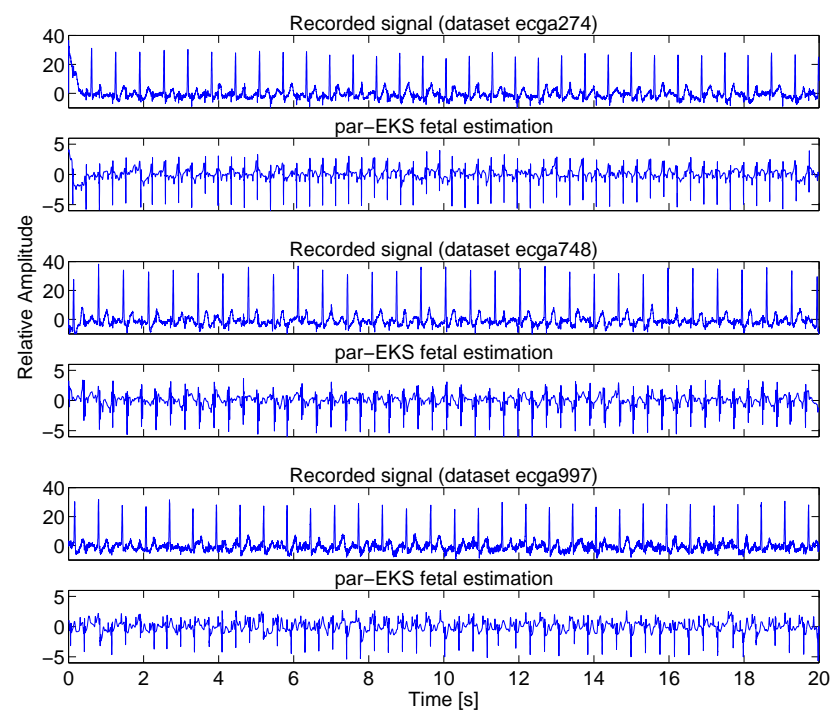

Figure 8. ECG mixtures of the datasets ecgca274 channel 5, ecgca748 channel 4, and ecgca997 channel 3 and their fetal par-EKS outputs.

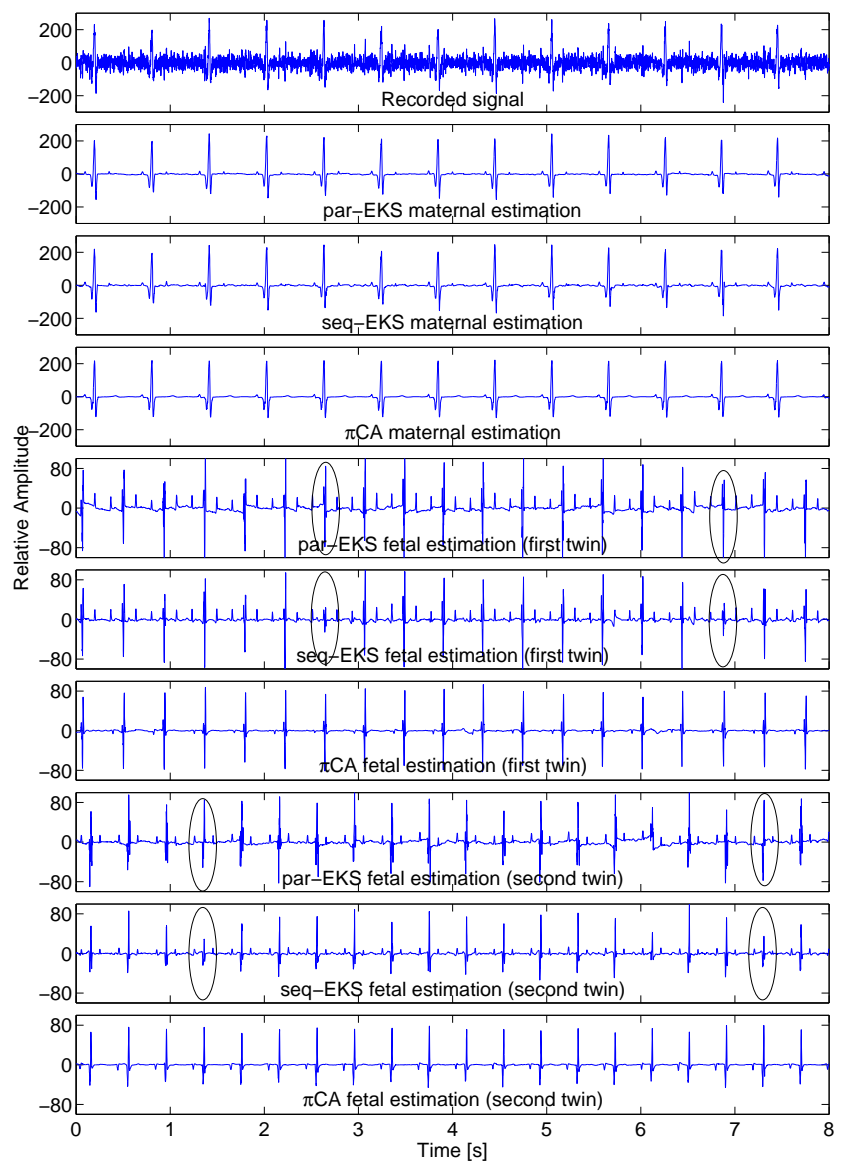

Figure 9. Results of the seq-EKS, par-EKS, and $\pi$ CA on twin MCG data. 


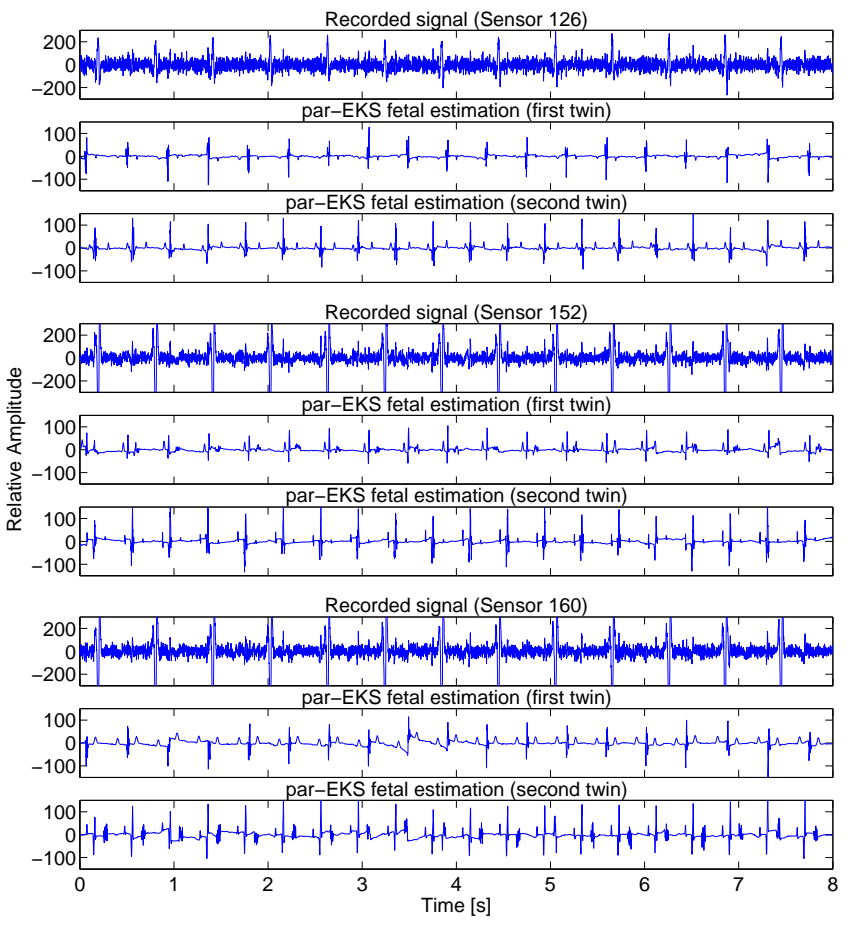

Figure 10. MCG mixtures of the channels 126, 152, and 160 and their fetal par-EKS outputs.

extract the two fetal MCG (fMCG) while seq-EKS fails to discriminate correctly the two fMCGs when they overlap (see highlighted signal parts, Fig. 9). In order to show the good behavior of par-EKS in several configurations, par-EKS is applied on other sensors (Fig. 10). One can note that the proposed par-EKS succeeds to extract the two fetal MCGs.

Finally, it is worth noting that the crucial part of the proposed par-EKS is the R-peaks detection. Although this detection is quite direct when a single fetus is present (Section [I-C), some words should be added on twin data. Indeed, on such data the detection of the mother's R-peaks is still direct since it is the dominant signal. On the contrary the discrimination between the two fetal R-peaks is much more difficult. Even though in this study, the oracle is obtained using several sensors and applying an ICA algorithm (here, we used Fast-ICA), it can be replaced in practice by a sound sensor located on the mother's abdomen.

\section{CONClusion}

In this paper, a synthetic dynamic ECG model within a KF framework has been extended to jointly model several ECGs to extract desired ECGs from a unique mixture (i.e. one channel recording) of maternal and fetal ECGs and noise. Although the proposed method only uses a single channel to separate different ECGs, since each ECG has a corresponding term in the model, the proposed model can efficiently discriminate ECGs even if desired and undesired ECG waves overlap in time. As proved on synthetic data and illustrated on actual data (single and multiple fetal pregnancy), the main merit of the proposed algorithm relies on its performance in a large class of situations. Performance of the proposed method on extraction of fECG from one mixture of $\mathrm{mECG}$ and fECG was examined according to noise level, amplitude ratio and heart rate ratio parameters: results show that the proposed method can be successfully employed in many scenarios. According to the obtained results, as long as R-peaks are correctly detected, the proposed model achieves good results. Although a reliable R-peaks detection is a straight forward procedure in a single fetal pregnancy (which most likely happens) even with a single sensor, it is much more difficult in multiple fetal pregnancy (twin or more). Nonetheless, in these situations, the R-peaks detection can be provided by other modalities such as echocardiography.

Finally, the proposed method compares favorably with efficient multi-sensor methods such as $\pi \mathrm{CA}$ (which also requires reliable R-peaks detection), while it requires only one sensor. The latter criterion is of high interest, since using a single channel does not only mean less electronic components (such as analog to digital converters or amplifiers) and thus a cheaper device, but also a more convenient and portable device for a long term monitoring system or at home since only a single electrode has to be placed on the mother's abdomen.

Perspectives include extension of the proposed method to apply on multichannel (but with a small number of channels, e.g., up to 3 or 4) mixtures of mECG and fECG. Moreover, synchronous echocardiography data can also be used in future works, especially for a reliable R-peaks detection.

\section{ACKNOWLEDGEMENT}

The authors would like to thank Dr. Reza Sameni for his support and assistance in this study, and Dr. Dirk Hoyer for providing the twin MCG dataset.

\section{REFERENCES}

[1] H. M. Jenkins, "Technical progress in fetal electrocardiography-a review.," J Perinat Med, vol. 14, no. 6, pp. 365-370, 1986.

[2] G. Camps, M. Martinez, and E. Soria, "Fetal ECG extraction using an fir neural network," in Proc. Computers in Cardiology 2001, 2001, pp. 249-252.

[3] R. Sameni and G. D. Clifford, "A Review of Fetal ECG Signal Processing; Issues and Promising Directions," The Open Pacing, Electrophysiology \& Therapy Journal (TOPETJ), vol. 3, pp. 4-20, 2010.

[4] A. Khamene and S. Negahdaripour, "A new method for the extraction of fetal ECG from the composite abdominal signal," IEEE Trans. Biomed. Eng., vol. 47, no. 4, pp. 507-516, 2000.

[5] P.P. Kanjilal, S. Palit, and G. Saha, "Fetal ECG extraction from singlechannel maternal ECG using singular value decomposition," IEEE Trans. Biomed. Eng., vol. 44, no. 1, pp. 51 -59, 1997.

[6] J.-F. Cardoso, "Multidimensional Independent Component Analysis," in Proc. of the IEEE International Conference on Acoustics, Speech, and Signal Processing (ICASSP '98), May 1998, vol. 4, pp. 1941-1944.

[7] L. de Lathauwer, B. de Moor, and J. Vandewalle, "Fetal electrocardiogram extraction by blind source subspace separation," IEEE Trans. Biomed. Eng., vol. 47, no. 5, pp. 567 -572, may 2000.

[8] R. Sameni, C. Jutten, and M. B. Shamsollahi, "Multichannel Electrocardiogram Decomposition using Periodic Component Analysis," "IEEE Trans. Biomed. Eng. ", vol. 55, no. 8, pp. 1935-1940, August 2008.

[9] J.L. Camargo-Olivares, R. Marti-Clemente, S. Hornillo-Mellado, M.M Elena, and I. Roman, "The maternal abdominal ECG as input to MICA in the fetal ECG extraction problem," Signal Processing Letters, IEEE, vol. 18 , no. 3, pp. $161-164$, march 2011.

[10] A. K. Barros and A. Cichocki, "Extraction of specific signals with temporal structure," Neural Computation, vol. 13, no. 9, pp. 1995-2003, Sept. 2001.

[11] Z. Yi Z. Zhang, "Extraction of temporally correlated sources with its application to non-invasive fetal electrocardiogram extraction," Neurocomputing, vol. 69, no. 7-9, pp. 894-899, 2006. 
[12] Y. Li and Z. Yi, "An algorithm for extracting fetal electrocardiogram," Neurocomputing, vol. 71, no. 7-9, pp. 1538 - 1542, 2008.

[13] V. Vigneron, A. Paraschiv-Ionescu, A. Azancot, O. Sibony, and C. Jutten, "Fetal electrocardiogram extraction based on non-stationary ICA and wavelet denoising," in Proc. Seventh Int Signal Processing and Its Applications Symp, 2003, vol. 2, pp. 69-72.

[14] M. G. Jafari and J. A. Chambers, "Fetal electrocardiogram extraction by sequential source separation in the wavelet domain," IEEE Trans. Biomed. Eng., vol. 52, no. 3, pp. 390-400, 2005.

[15] R. Martin-Clemente, J.L. Camargo-Olivares, S. Hornillo-Mellado, M. Elena, and I. Roman, "Fast technique for noninvasive fetal ECG extraction," IEEE Trans. Biomed. Eng., vol. 58, no. 2, pp. 227-230, 2011.

[16] R. Sameni, Extraction of Fetal Cardiac Signals from an Array of Maternal Abdominal Recordings, Ph.D. thesis, Sharif University of Technology - Institut National Polytechnique de Grenoble, July 2008, Available Online: http://www.sameni.info/Publications/Thesis/PhDThesis.pdf

[17] M. Kotas, "Projective filtering of time-aligned ECG beats," IEEE Trans. Biomed. Eng., vol. 51, no. 7, pp. 1129-1139, 2004.

[18] B. Widrow, Jr. Glover, J.R., J.M. McCool, J. Kaunitz, C.S. Williams, R.H. Hearn, J.R. Zeidler, Jr. Eugene Dong, and R.C. Goodlin, "Adaptive noise cancelling: Principles and applications," Proceedings of the IEEE, vol. 63, no. 12, pp. $1692-1716,1975$.

[19] N.J. Outram, E.C. Ifeachor, P.W.J. Van Eetvelt, and J.S.H. Curnow, "Techniques for optimal enhancement and feature extraction of fetal electrocardiogram," Science, Measurement and Technology, IEE Proceedings -, vol. 142 , no. 6, pp. $482-489,1995$.

[20] A. G. Favret, "Computer matched filter location of fetal R-waves.," Med Biol Eng, vol. 6, no. 5, pp. 467-475, Sep 1968.

[21] Y. C. Park, K. Y. Lee, D. H. Youn, N. H. Kim, W. K. Kim, and S. H. Park, "On detecting the presence of fetal R-wave using the moving averaged magnitude difference algorithm," IEEE Trans. Biomed. Eng. vol. 39, no. 8, pp. 868-871, 1992.

[22] V. Zarzoso and A. K. Nandi, "Noninvasive fetal electrocardiogram extraction: blind separation versus adaptive noise cancellation," IEEE Trans. Biomed. Eng., vol. 48, no. 1, pp. 12-18, 2001.

[23] R. Sameni, M. B. Shamsollahi, C. Jutten, and G. D. Clifford, "A Nonlinear Bayesian Filtering Framework for ECG Denoising," IEEE Trans. Biomed. Eng., vol. 54, no. 12, pp. 2172-2185, December 2007.

[24] G. Welch and G. Bishop, "An introduction to the Kalman filter," Tech. Rep., Chapel Hill, NC, USA, 1995.

[25] P.E. McSharry, G.D. Clifford, L. Tarassenko, and L.A. Smith, "A dynamical model for generating synthetic electrocardiogram signals," IEEE Trans. Biomed. Eng., vol. 50, no. 3, pp. 289 -294, 2003.

[26] G. D. Clifford, A. Shoeb, P. E. McSharry, and B. A. Janz, "Modelbased filtering, compression and classification of the ECG," Int. J. Bioelectromag., vol. 7, no. 1, pp. 158-161, 2005.

[27] R. Sameni, G. D. Clifford, C. Jutten and M. B. Shamsollahi, "Multichannel ECG and Noise Modeling: Application to Maternal and Fetal ECG Signals," EURASIP Journal on Advances in Signal Processing, vol. 2007, pp. Article ID 43407, 2007.

[28] T. J. DuBose, J. A. Cunyus, and L. F. Johnson, "Embryonic heart rate and age," Journal of Diagnostic Medical Sonography, vol. 6, no. 3, pp. 151-157, 1990.

[29] B. De Moor, P. De Gersem, B. De Schutter, and W. Favoreel, "DAISY: A database for identification of systems," Journal A, Special Issue on CACSD (Computer Aided Control Systems Design), vol. 38, no. 3, pp. 4-5, Sept. 1997.

[30] A. L. Goldberger, L. A. N. Amaral, L. Glass, J. M. Hausdorff, P. Ch. Ivanov, R. G. Mark, J. E. Mietus, G. B. Moody, C.-K. Peng, and H. E. Stanley, "PhysioBank, PhysioToolkit, and PhysioNet: Components of a new research resource for complex physiologic signals," Circulation, vol. 101, no. 23, pp. e215-e220, 2000 (June 13), Circulation Electronic Pages: http://circ.ahajournals.org/cgi/content/full/101/23/e215.

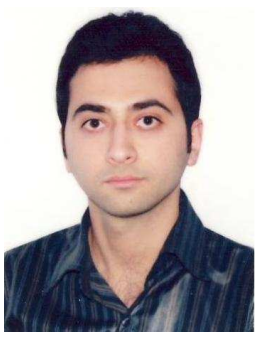

Mohammad Niknazar was born in Tehran, Iran in 1985. He received his B.Sc. degree in electricalelectronic engineering from the University of Semnan, Iran in 2007. He received his M.Sc. degree in electrical-biomedical engineering from Sharif University of Technology, Tehran, Iran, in 2010. As a member of GIPSA-lab, he is currently working towards the Ph.D. degree in signal, image and speech processing and telecommunications at Joseph Fourier University, Grenoble, France.

His research interests include signal and image processing, nonlinear (chaos) analysis, blind source separation, and pattern recognition. Current areas of application include fetal ECG extraction and ECG processing. Previous areas of application include epileptic seizure detection and prediction.

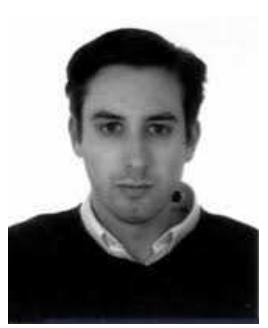

Bertrand Rivet was graduated from the École Normale Supérieure de Cachan, France. He received the Agrégation de Physique Appliquée in 2002, the Master's degree from the University of Paris-XI, France, in 2003 and the $\mathrm{PhD}$ degree from Grenoble Institute of Technology (GIT), France, in 2006.

$\mathrm{He}$ is currently an Associate Professor in signal processing with PHELMA and a member of GIPSAlab, GIT, France. His research concerns biomedical signal processing, audiovisual speech processing and blind source separation.

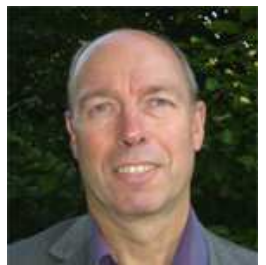

Christian Jutten received the $\mathrm{PhD}$ degree in 1981 and the Docteur s Sciences degree in 1987 from the Institut National Polytechnique of Grenoble (France). He taught as associate professor in the Electrical Engineering Department from 1982 to 1989, before to become full professor in University Joseph Fourier of Grenoble. He was visiting professor in Swiss Federal Polytechnic Institute in Lausanne in 1989 and in Campinas University (Brazil) in 2010. He has been deputy director of the Grenoble images, speech, signal and control laboratory (GIPSA, 300 people) and director of the Department Images-Signal (DIS, 100 people) from 2007 to 2010. For 30 years, his research interests are blind source separation, independent component analysis and learning in neural networks, including theoretical aspects (separability, source separation in nonlinear mixtures, sparsity) and applications in signal processing (biomedical, seismic, hyperspectral imaging, speech). He is author or co-author of more than 65 papers in international journals, 4 books, 22 invited plenary talks and 150 communications in international conferences. He has been associate editor of IEEE Trans. on Circuits and Systems (1994-95), and co-organizer the 1st International Conference on Blind Signal Separation and Independent Component Analysis (Aussois, France, January 1999). He has been a scientific advisor for signal and images processing at the French Ministry of Research from 1996 to 1998 and for the French National Research Center (CNRS) from 2003 to 2006 . He is currently deputy director of Institute for Information Sciences and Technologies of CNRS. He received the Medal Blondel in 1997 from SEE (French Electrical Engineering society) for his contributions in source separation and independent component analysis, and has been elevated as a Fellow IEEE and a senior Member of Institut Universitaire de France in 2008. 